\title{
Double pass remotely pumped L-band EDFA with the integration of Raman amplification using multiwavelength dispersion compensator
}

\begin{abstract}
We demonstrate a remotely pumped L-band erbium-doped fiber amplifier for long-haul transmission system that consists of a multiwavelength dispersion compensator. In this proposed amplifier, the L-band signals experience double pass amplification using the multiwavelength dispersion compensator as signal reflector. In addition, the distributed Raman amplification helps to improve the gain flatness of the propagated signals that is pumped by a $1497 \mathrm{~nm}$ fiber laser. High average transmission gain of $20.64 \mathrm{~dB}$ with low gain flatness of $2.64 \mathrm{~dB}$ is attained when the input signal power is at $0 \mathrm{~dB}$ m with wavelength ranging from 1570.45 to $1604.92 \mathrm{~nm}$. The proposed amplifier structure offers better transmission gain flatness for multiwavelength systems.
\end{abstract}

Keyword: Laser amplifiers; Erbium-doped fiber amplifier; Fiber lasers; Fiber non-linear optics 